\title{
Improvement of cardiac function in the failing rat heart after transfer of skeletal myoblasts engineered to overexpress placental growth factor
}

\author{
Matthias Gmeiner, MD, ${ }^{\mathrm{a}}$ Daniel Zimpfer, MD, ${ }^{\mathrm{b}}$ Johannes Holfeld, MD, ${ }^{\mathrm{b}}$ Gernot Seebacher, MD, ${ }^{\mathrm{b}}$ \\ Dietmar Abraham, PhD, ${ }^{a}$ Michael Grimm, MD, ${ }^{\mathrm{b}}$ and Seyedhossein Aharinejad, MD, PhD ${ }^{\mathrm{a}, \mathrm{b}}$
}

\begin{abstract}
Background: Transplant of skeletal myoblasts is an attractive alternative to repair irreversibly damaged myocardium in ischemic heart failure. We investigated whether transplant of myoblasts overexpressing placental growth factor would stimulate angiogenesis and enhance myoblast survival in a rat heart failure model.
\end{abstract}

\begin{abstract}
Methods: Three weeks after myocardial infarction, Sprague-Dawley rats in heart failure received intramyocardial injections of Ringer solution (control) or autologous myoblasts, unmodified or transfected with placental growth factor expression plasmid. Sham-operated animals served as noninfarct controls. Cardiac function was assessed by echocardiography to 86 days after engraftment. Immunocytochemistry and fluorescence imaging were used to investigate vessel formation, grafted myoblast survival, infarct wall thickness, and infarct size. Quantitative real-time reverse transcriptase polymerase chain reaction and Western blotting measured tissue messenger RNA and protein expressions.
\end{abstract}

Results: Left ventricular function significantly improved with time, and fractional shortening on day 86 was significantly enhanced in transfected myoblast group relative to control $(P<.01)$ and unmodified myoblast $(P<.05)$ groups. Vascular density $(P<.01)$ and myoblast survival $(P<.05)$ were enhanced in rats treated with transfected myoblasts relative to other groups $(P<.05)$. Mean fraction of fibrotic scar tissue was decreased in unmodified and transfected myoblast groups relative to controls on day $86(P<.05)$, and left ventricular wall thickness was significantly increased in transfected myoblast group relative to other groups $(P<.05)$.

Conclusions: Intramyocardial injections of autologous myoblasts overexpressing placental growth factor improved cardiac function, attenuated adverse cardiac remodeling, induced angiogenesis, and probably enhanced survival of grafted myoblasts. (J Thorac Cardiovasc Surg 2011;141:1238-45)

Heart failure resulting from myocardial infarction is growing in incidence, and heart transplant is the only accepted treatment for end-stage patients. Because of organ shortage, however, such alternative approaches as transplant of autologous skeletal myoblasts have received tremendous interest in experimental and human studies. ${ }^{1-4}$ However, the MAGIC (Myoblast Autologous Grafting in Ischemic Cardiomyopathy) trial ${ }^{5}$ failed to improve cardiac function in patients although remodeling of the myocardium was ameliorated. Earlier reports have indicated that the outcome of myoblast transplant is dependent on the number of grafted cells $\mathrm{s}^{3}$ and enhancing myoblast survival therefore

From the Laboratory for Cardiovascular Research, ${ }^{\mathrm{a}}$ Center for Anatomy and Cell Biology, and the Department of Cardiac Surgery, ${ }^{\mathrm{b}}$ Medical University of Vienna, Vienna, Austria.

Supported by grant No. 2465 from the Medical Foundation of the Mayor of Vienna (to M. Grimm and S.A.) and by the CARDIOWORKBENCH EU-grant No. PL 018671 (to S.A.).

Disclosures: Authors have nothing to disclose with regard to commercial support.

Received for publication July 24, 2009; revisions received Oct 4, 2010; accepted for publication Oct 23, 2010; available ahead of print Feb 17, 2011.

Address for reprints: Seyedhossein Aharinejad, MD, PhD, Department of Cardiac Surgery, Medical University of Vienna, Waehringerguertel 18-20, A-1090 Vienna, Austria (E-mail: seyedhossein.aharinejad@meduniwien.ac.at).

0022-5223/\$36.00

Copyright (c) 2011 by The American Association for Thoracic Surgery doi:10.1016/j.jtcvs.2010.10.054 might be beneficial. Accordingly, experimental studies have attempted to improve myoblast graft survival by using colony-stimulating factor 1 , hypoxia inducible factor, and vascular endothelial growth factor (VEGF). ${ }^{6-8}$

Placental growth factor (PIGF) is a VEGF homolog that is predominantly expressed in the placenta, heart, and lung. ${ }^{9}$ Although VEGF and PIGF have synergistic effects, ${ }^{10}$ PIGF binds exclusively to the fms-like tyrosine kinase (FLT1) receptor and the neuropilin 1 coreceptor. ${ }^{9}$ PlGF is proangiogenic and induces chemotactic, proliferative, and survival signals for cells reportedly involved in arteriogenesis, such as endothelial cells, smooth muscle cells, and monocytes. $^{11,12}$ Whether myoblasts engineered to overexpress $\mathrm{PlGF}$ could improve cardiac function is unknown, so we addressed this issue.

\section{MATERIALS AND METHODS}

\section{Experimental Design and Animals}

All experiments were approved by the ethics committee of the Medical University of Vienna, and the investigation conformed to the Guide for the Care and Use of Laboratory Animals published by the US National Institute of Health (NIH Publication No. 85-23, revised 1996; www.nap.edu/ catalog/5140.html). A total of 95 Sprague-Dawley rats (240-280 g, 8-10 weeks old) were used (Harlan Laboratories, Inc, Boxmeer, The Netherlands). Myocardial infarction was induced in 75 anesthetized animals, ${ }^{6}$ 


\section{Abbreviations and Acronyms \\ $\alpha$-SMA $=\alpha$-smooth muscle actin \\ DiIC $_{18}=1,1^{\prime}$-dioctadecyl-3,3, $3^{\prime}, 3^{\prime}$ - tetramethylindocarbocyanine perchlorate \\ $\mathrm{EF} \quad=$ ejection fraction \\ FLT1 = fms-like tyrosine kinase \\ FS $\quad=$ fractional shortening \\ KDR $=$ kinase insert domain receptor \\ $\mathrm{LV}=$ left ventricular \\ LVEDD $=$ left ventricular end-diastolic diameter \\ LVESD = left ventricular end-systolic diameter \\ MB = unmodified myoblast transplant [group] \\ MB-PlGF $=$ myoblast overexpressing placental growth factor transplant [group] \\ MMP = matrix metalloproteinase \\ PlGF = placental growth factor \\ TIMP $=$ tissue inhibitor of matrix metalloproteinase \\ VEGF $=$ vascular endothelial growth factor \\ $\mathrm{vWF}=$ von Willebrand factor}

and cardiac function was assessed in surviving animals $(n=45)$ by echocardiography on day 21 . The rats with comparable fractional shortening (FS) were divided into 3 groups of 15 ; they then underwent rethoracotomy, and $150 \mu \mathrm{L}$ Dulbecco modified Eagle medium containing $5 \times 10^{6}$ myoblasts labeled with $1,1^{\prime}$-dioctadecyl-3,3,3',3'-tetramethylindocarbocyanine perchlorate $\left(\mathrm{DiIC}_{18}\right)$ or $150 \mu \mathrm{L}$ Ringer solution were injected into the infarcted myocardium with a 30 -gauge needle. ${ }^{6}$ The volume delivered was evenly divided between the central ( 2 injections) and bordering ( 5 injections) areas of the infarcted myocardium. Animals were treated by intramyocardial injection of $5 \times 10^{6}$ autologous myoblasts overexpressing rat PIGF (MB-PlGF group), unmodified myoblasts (MB group) or Ringer solution (control group). Sham-operated rats $(\mathrm{n}=20)$ served as additional noninfarct controls (sham group). On days 52 and 86, cardiac function was reassessed.

\section{Plasmid Construction, Myoblast Culture, and Transfection}

The 477-base pair rat PIGF open reading frame ${ }^{13}$ was amplified by polymerase chain reaction from an Image clone (7193034; ATTC 10467199, Genbank BC087006, CK603317) with 5'-AAGATGCTGGC CATGAAGCT- $3^{\prime}$ forward and $5^{\prime}$-TCACAGGTGGGGTTCCTCAG-3' reverse primers with high-fidelity platinum $\mathrm{Taq}$ polymerase (Invitrogen $\mathrm{GmbH}$, Lofer, Austria). The polymerase chain reaction product was separated on 1\% agarose gels by electrophoresis, purified with Qiaex II (QIAGEN GmbH, Hilden, Germany), and topoisomerase I-mediated TA cloning cloned under control of the cytomegalovirus immediate early promoter into pcDNA2.1/V5-His-TOPO (Invitrogen) as p(r)PIGF. The reverse primer design maintained the natural PlGF stop codon to prevent translation of the V5 epitope in the expression construct. Plasmids were prepared with Genelute (Sigma, St Louis, Mo) maxipreps, and the inserts were confirmed by sequencing with a T7 primer on an ABI 3100 genetic analyzer (Life Technologies Corporation, Carlsbad, Calif).

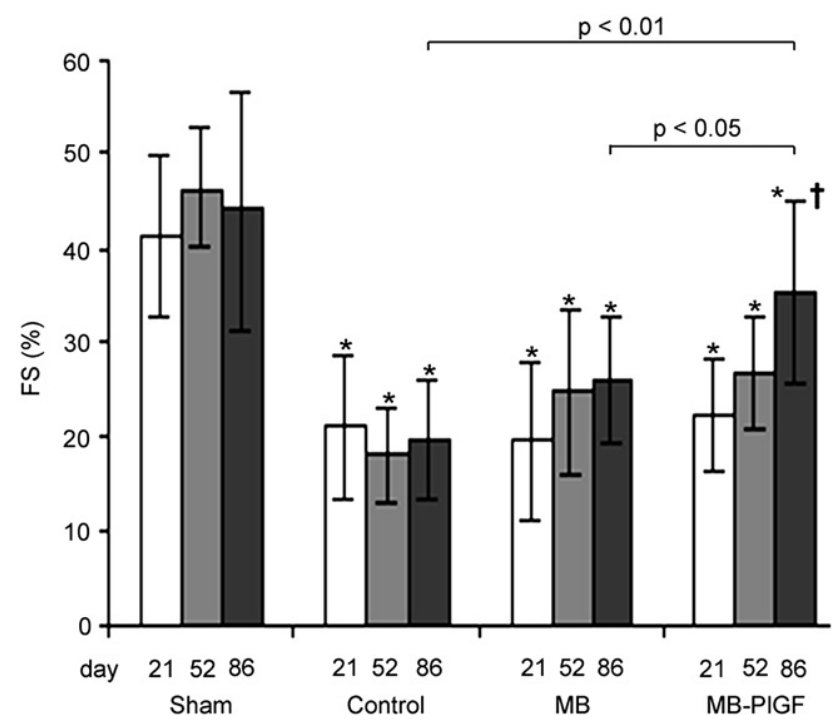

FIGURE 1. Left ventricular function. Only group treated with myoblasts transfected with placental growth factor $(M B-P l G F)$ showed improved fractional shortening $(F S)$ significantly with time, whereas in group treated with unmodified myoblasts $(M B)$ and control group, fractional shortening values on days 52 and 86 were not significantly different from values on day 21. In all infarct groups, fractional shortening was lower than in sham groups. Fractional shortening on day 86 in group with transfected myoblasts was higher than fractional shortening on day 86 in group with unmodified myoblasts $(P<.05)$ and control group $(P<.01)$. Asterisk indicates significant difference from sham group $(P<.05)$. Dagger indicates significant differences on days 21 and 52 versus day $86(P<.05)$.

Before myocardial infarction was induced, the right anterior tibial muscle was isolated, minced in phosphate-buffered saline solution, and passed through a $100-\mu \mathrm{m}$ sieve (BD, Franklin Lakes, NJ). Cells were cultured in Dulbecco modified Eagle medium supplemented with $10 \%$ fetal calf serum, ${ }^{6}$ screened with myocyte-specific antidesmin (Lab Vision/ NeoMarkers, Fremont, Calif) and fibroblast-specific antibody (Harlan Sera-Lab Ltd, Loughborough, UK), and transfected with the p(r)PlGF or empty vector with Effecten transfection reagent (QIAGEN). Transfection efficiency was determined ${ }^{6}$ and was approximately $70 \%$.

\section{Echocardiography and Electrocardiography}

Rats were anesthetized with isoflurane, and the maximal left ventricular (LV) long-axis length and endocardial area tracing were measured with a $15-\mathrm{MHz}$ linear array scan head to calculate LV end-diastolic diameter (LVEDD), LV end-systolic diameter (LVESD), end-diastolic volume (LVEDV), and end-systolic volume (LVESV). ${ }^{6}$ LV FS and ejection fraction (EF) were calculated according to the following formulas: FS $=[($ LVEDDLVESD $/$ LVEDD $] \times 100 \%$ and $\mathrm{EF}=[($ LVEDV - LVESV $) /$ LVEDV] $\times 100 \%$. Measurements used 3 consecutive cardiac cycles. A telemetry device (Data Sciences International, Minneapolis, Minn) recorded electrocardiographic signals continuously ${ }^{6}$ (http://cardiovascres.oxford journals.org/content/79/3/395.long).

\section{Histology}

Tissue was processed for paraffin and cryosectioning embedding. ${ }^{6} \mathrm{Se}-$ rial sections were stained with hematoxylin-eosin and Goldner trichrome for evaluation of myocardial scar fibrosis and LV infarct wall thickness. For histologic quantification of DiIC 18 -labeled myoblasts, serial heart sections were counterstained with 4,6-diamidino-2-phenylindole (Molecular Probes, Inc, Eugene, Ore) and embedded in AF1 antifading agent (Citifluor 
TABLE 1. Echocardiographic data on day 86

\begin{tabular}{lccc}
\hline \multicolumn{1}{c}{ Parameter } & Control & MB & MB-PIGF \\
\hline LVEDD $(\mathrm{cm})$ & $0.8 \pm 0.13$ & $0.68 \pm 0.08$ & $0.65 \pm 0.15^{*}$ \\
LVESD $(\mathrm{cm})$ & $0.61 \pm 0.14$ & $0.5 \pm 0.09$ & $0.4 \pm 0.1 \dagger \neq$ \\
LVEDV $(\mathrm{mL})$ & $1.7 \pm 0.2$ & $1.6 \pm 0.1$ & $1.4 \pm 0.2 *$ \\
LVESV $(\mathrm{mL})$ & $1.4 \pm 0.3$ & $1.2 \pm 0.2$ & $1 \pm 0.1 \dagger \neq$ \\
Ejection fraction $(\%)$ & $22 \pm 7$ & $24 \pm 8$ & $34 \pm 6 \S$ \\
\hline
\end{tabular}

$M B$, Unmodified myoblast group; $M B-P l G F$, myoblast group overexpressing placental growth factor; $L V E D D$, left ventricular end-diastolic diameter; $L V E S D$, left ventricular end-systolic diameter; $L V E D V$, left ventricular end-diastolic volume; LVESV, left ventricular end-systolic volume. ${ }^{*} P<.05$ versus control group. $\dagger P<.01$ versus control group. $\ddagger P<.05$ versus unmodified myoblast group. $\S P<.01$ versus control and unmodified myoblast groups.

Ltd, Leicester, UK). For immunohistochemistry, deparaffinized sections were incubated with $3 \%$ hydrogen peroxide, blocked in $5 \%$ horse serum (Jackson ImmunoResearch Laboratories, Inc, West Grove, Pa) and then incubated with a polyclonal anti-rat von Willebrand factor (vWF; Abcam, Cambridge, UK) or a monoclonal anti- $\alpha$-smooth muscle actin ( $\alpha$-SMA; Sigma) antibody. Primary antibodies were detected with appropriate biotinylated secondary antibodies (Vector Laboratories, Inc, Burlingame, Calif) and peroxidase-conjugated streptavidin (Dako North America, Inc, Carpinteria, Calif) developed with 3,3'-diaminobenzidine (Vector Laboratories), and counterstained with hematoxylin. For quantitative histologic analysis, digitized images were made at $40 \times$ magnification. Quantification was performed with Lucia G image analysis software (Lucia G; OPTOTEAM Präzisionsinstrumente, Vienna, Austria). Three randomly chosen fields of the myocardial wall in the region of the anterior branch of the left coronary artery were evaluated in each heart. Vascular density, vWF-positive cells, and $\alpha$-SMA positive cells were all evaluated.

\section{Fluorescence Optical Imaging}

In experiments with $\mathrm{DiIC}_{18}$-labeled myoblasts, 3-dimensional cellular integration was assessed on an imaging station for all animals (Kodak
IS2000MM; Eastman Kodak Company, New Haven, Conn). Ventricles were then dissected in 5 animals per group and mechanically dissociated in collagenase, and the percentage of grafted DiIC $_{18}$-labeled myoblasts was detected with an inverted fluorescent microscope.

\section{Real-Time Reverse Transcriptase Polymerase Chain Reaction Analysis}

Real-time reverse transcriptase polymerase chain reaction analysis was performed as described. ${ }^{6}$ The primer sequences (sense/antisense) were as follows: PIGF, 5'-ACTGTGTGGCGCTAAAGACA-3'/5'-TTCCTCAGTCTG TGGGGTTT-3'; VEGF-A, 5'-TCCTGCAGCATAGCAGATGT-3'/5'-GCG AGTCTGTGTTTTTGCAG-3'; kinase insert domain receptor (KDR), $5^{\prime}$ GGAGATTGAAAGAAGGAAC-3' $3^{\prime}$ - ACTTCCTCTTCCTCCATAC- $3^{\prime}$; FLT1, 5'-GGAGGCGAGGATTACAGTGA-3'/5'-GGAGGCGAGGATTA CAGTGA-3'; $\beta_{2}$-microglobulin (as a housekeeping gene), $5^{\prime}$-ACTGCT ACGTGTCTCAGTTC-3'/5'-CAAGCATATACATCGGTCTC-3' .

\section{Western Blotting}

Western blots were probed with polyclonal antibodies against PIGF (Santa Cruz Biotechnology, Inc, Santa Cruz, Calif), VEGF-A (Thermo Fisher Scientific, Waltham, Mass), matrix metalloproteinases (MMPs) 2, and 9, tissue inhibitors of MMP (TIMPs) 1, 2, 3, and 4 (Millipore Corporation, Billerica, Mass). ${ }^{6}$

\section{Statistical Analysis}

Data were compared between the groups with the Wilcoxon rank test and analysis of variance with the Bonferroni $t$ test after testing for normality of distribution by Kolmogorov-Smirnov test. The echocardiographic data were subjected to repeated-measures analysis of variance by using the repeated statement in the mixed procedure. Post hoc comparisons were performed with the Tukey-Kramer method of adjustment for multiple comparisons. Statistical analyses were performed with the SAS software system for Windows version 9.1.3 and Enterprise Guide version 4.1 (SAS Institute, Inc, Cary, NC). Data are expressed as mean \pm SD.

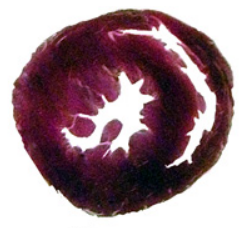

A

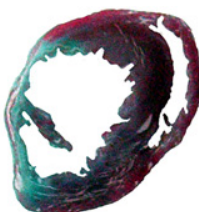

Control

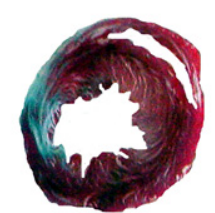

MB

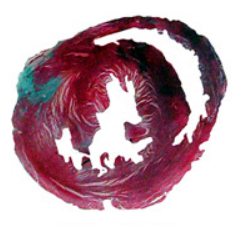

MB-PIGF
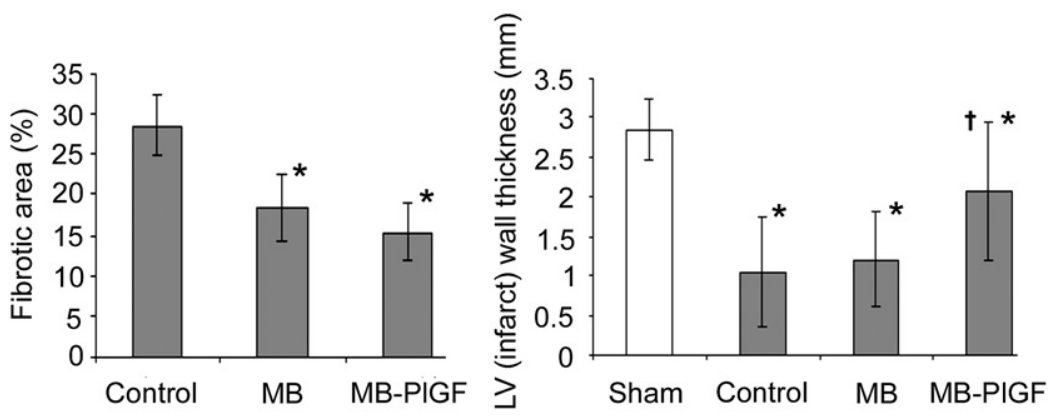

B

C

FIGURE 2. Cardiac remodeling in infarcted hearts. Representative histologic images (A) and quantitative morphometric analysis (B and C). B, Mean proportion of fibrotic tissue to normal left ventricular (LV) myocardium is decreased in unmodified myoblast group $(M B)$ and in placental growth factor-transfected myoblast group $(M B-P l G F)$ relative to control group. Asterisk indicates significant difference from control group $(P<.05)$. C, Left ventricular infarct wall thickness is increased in transfected myoblast group relative to unmodified myoblast and control groups. Asterisk indicates significant difference from sham group. Dagger indicates significant differences from unmodified myoblast and control groups $(P<.05)$. 


\section{RESULTS}

\section{Transplant of Myoblasts Overexpressing PIGF Improves LV Function}

Three weeks after myocardial infarction, FS was comparable among all treatment groups and significantly decreased relative to the sham group. FS was significantly improved on days $52(P<.05)$ and $86(P<.05)$ relative to day 21 in the MB-PlGF group but was not statistically different within the MB and control groups (Figure 1). In all experimental groups, the FS was significantly lower than in sham-operated animals on day 86 . On day $86, \mathrm{FS}$ in the MB-PlGF group was significantly higher than FS values on day 86 in the $\mathrm{MB}(P<.05)$ and control $(P<.01)$ groups

On day 86, LVEDV and LVEDD were reduced in the MBPlGF group relative to the control group $(P<.05)$; so were LVESV and LVESD relative to both the control group $(P<.01)$ and the MB group $(P<.05)$. Consequently, EF was higher in the MB-PlGF group than in both the MB and control groups $(P<.01$; Table 1$)$. Electrocardiographic recordings revealed ventricular arrhythmias in both the $\mathrm{MB}$ and MB-PIGF groups after myoblast engraftment, but these were self-limiting and hemodynamically well tolerated. These results clearly demonstrate that the transplant of myoblasts engineered to overexpress PlGF improves longterm cardiac function.

\section{Transplant of Myoblasts Overexpressing PIGF Attenuates Cardiac Remodeling}

Morphometric analysis revealed that the mean fraction of fibrotic scar tissue to normal LV myocardium was decreased in the MB and MB-PIGF groups relative to the control group when killed on day $86(P<.05$; Figure $2, A$ and $B)$. The LV wall thickness, however, was significantly increased in the MB-PlGF group relative to both the MB and control groups $(P<.05$; Figure $2, C)$. All experimental groups had a significantly reduced wall thickness relative to the sham group. These results further indicate that PIGF ameliorates cardiac remodeling associated with ischemiainduced heart failure.

\section{Fluorescent Myoblast Survival}

Shown representatively in Figure 3, A, fluorescent myoblasts in the anterior LV wall were seen in the MBPlGF and MB groups. Analysis of serial sections by fluorescence microscopy confirmed these data and revealed that fluorescent myoblasts were visible within the myocardial tissue in both groups (Figure 3, B). Ventricles were mechanically dissociated in collagenase, and the percentage of grafted DiIC 18 -labeled myoblasts was determined with an inverted fluorescent microscope. More grafted, fluorescent, DiIC $_{18}$-labelled myoblasts were seen in the MB-PIGF group than in the MB group $(P<0.05$; Figure 3,C).
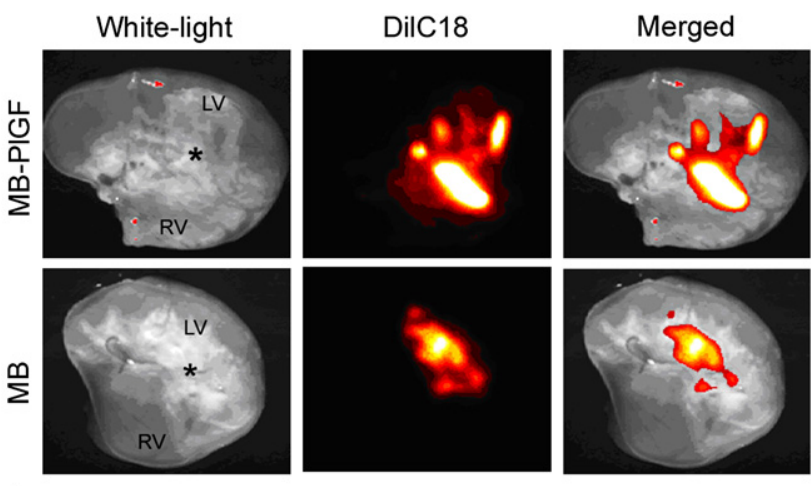

$\mathbf{A}$
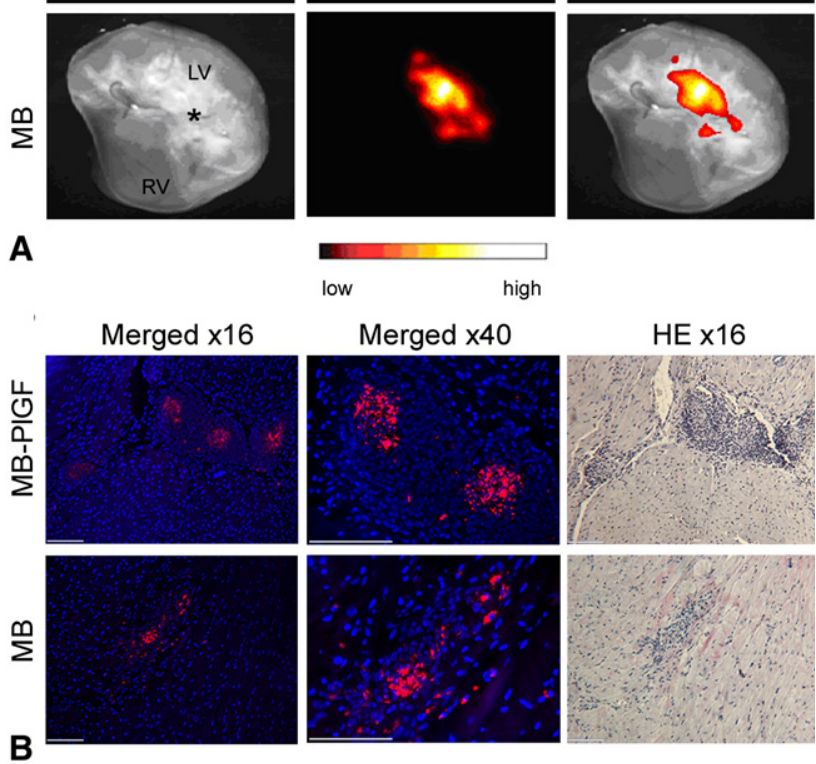

low

Merged $\times 40$
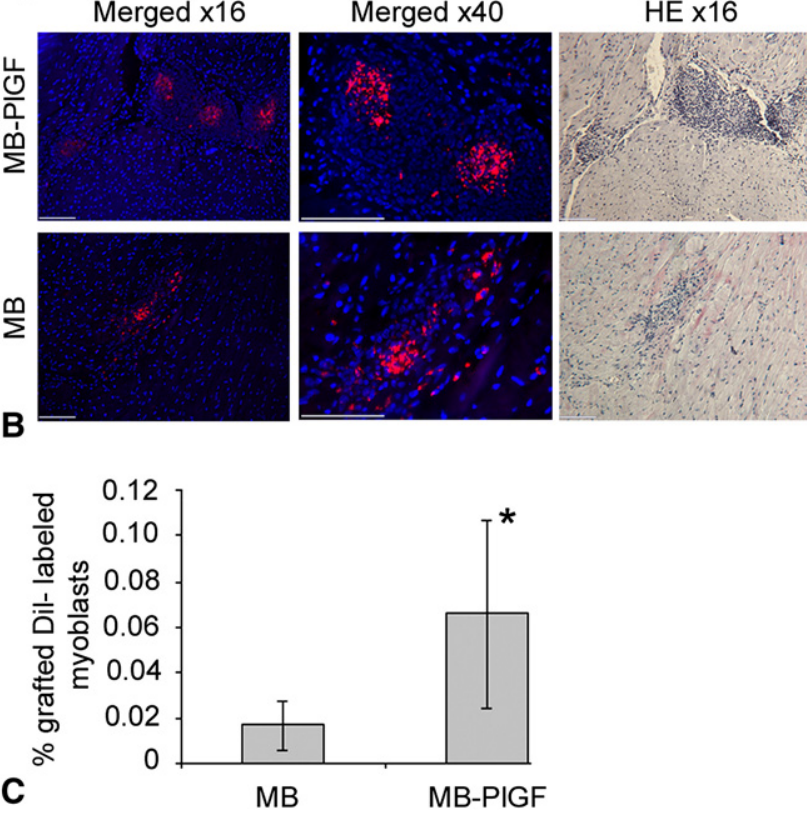

FIGURE 3. Fluorescent autologous myoblasts. A, Localization of myoblasts labeled with 1,1'-dioctadecyl-3,3,3',3'-tetramethylindocarbocyanine perchlorate, both unmodified $(M B)$ and overexpressing placental growth factor $(M B-P l G F) 86$ days after myocardial infarction. B, Representative white-light images (left), fluorescent $1,1^{\prime}$-dioctadecyl-3,3,3',3'-tetramethylindocarbocyanine perchlorate (DiIC18) signals (center), and merged images (right) showing cellular localization (red) in infarcted area of heart (asterisk). $L V$, Left ventricle; $R V$, right ventricle. Atria are removed. B, Representative red-channel fluorescence images of $1,1^{\prime}$-dioctadecyl-3,3, $3^{\prime}, 3^{\prime}$ tetramethylindocarbocyanine perchlorate signal merged with corresponding section counterstained with 4,6-diamidino-2-phenylindole in border zone of unmodified $(M B)$ and transfected $(M B-P l G F)$ myoblast groups. $B a r$ represents $50 \mu \mathrm{m}$. HE, Hematoxylin-eosin. C, Analyses revealed significantly more grafted fluorescent myoblasts labeled with $1,1^{\prime}$-dioctadecyl-3,3,3',3'-tetramethylindocarbocyanine perchlorate (DiI) in transfected myoblast group $(M B-P l G F)$ relative to unmodified myoblast $(M B)$ group in tissue lysates. Asterisk indicates significant difference from unmodified myoblast group $(P<.05)$.

\section{PIGF Enhances Angiogenesis}

Histologic analysis in the border zone of the infarct area revealed a significantly increased number of vWF-positive 


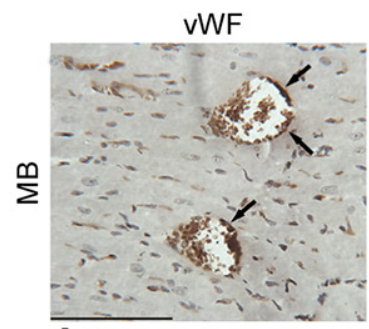

\section{A}
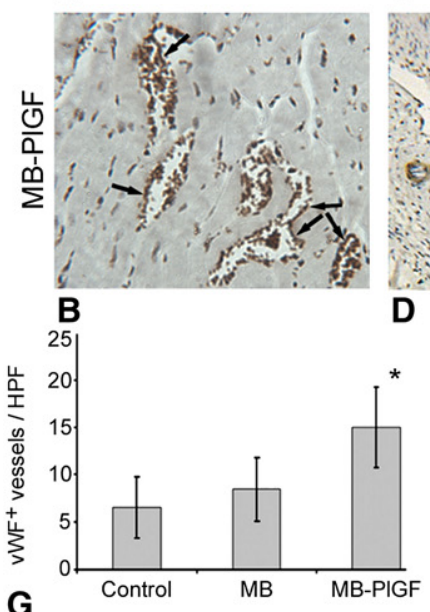

G

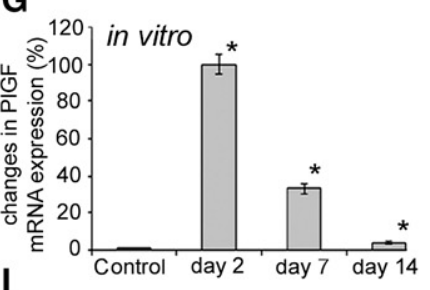

a SMA

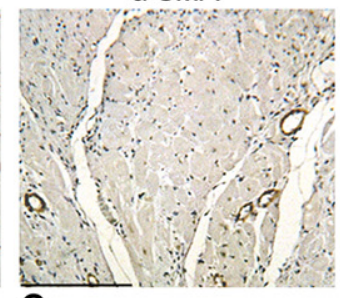

C

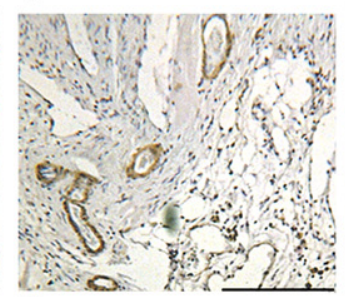

D

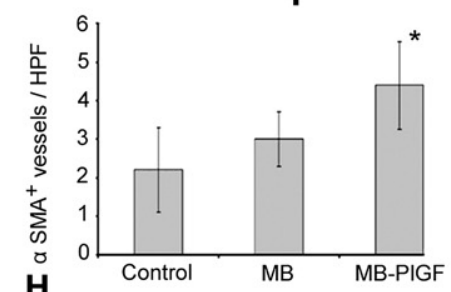

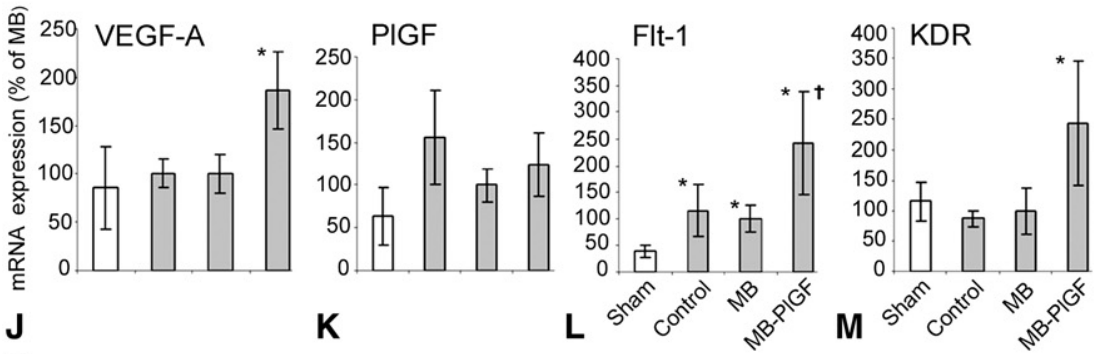

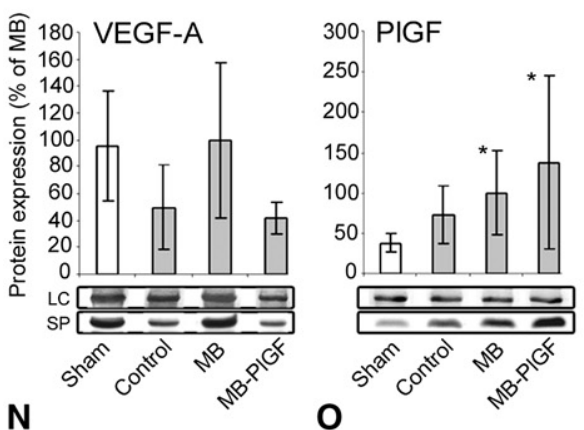

FIGURE 4. Analysis of angiogenesis. Representative sections stained for von Willebrand factor $(v W F)$ in (A) unmodified myoblasts $(M B)$ and $(B)$ placental growth factor-transfected myoblasts $(M B$-PlGF). Representative sections stained for $\alpha$-smooth muscle actin $(\alpha S M A)$ in unmodified myoblast $(\mathrm{C}$, E) and transfected myoblast (D, F) groups. Quantification of von Willebrand factor-positive $\left(v W F^{+}, \mathrm{G}\right)$ or $\alpha$-smooth muscle actin-positive $\left(\alpha S M A^{+}, \mathrm{H}\right)$ vessels 86 days after infarction (count per high-power field, $H P F$ ) showing increased vessel density in transfected myoblast group. Asterisk indicates significant 
vessels in the MB-PIGF group relative to both the MB and control groups $(P<.01$; Figure $4, A, B$, and $G) 86$ days after myocardial infarction. Furthermore, the number of $\alpha$-SMA-positive vessels was increased in the MB-PIGF group relative to both the $\mathrm{MB}$ and control groups $(P<.01$; Figure $4, C-F$ and $H)$. Transfection efficiency in cultured myoblasts was approximately $70 \%$. PIGF messenger RNA was overexpressed in vitro on days 2,7 , and 14 relative to expression in untransfected control myoblasts (Figure 4, I).

In line with these findings, we observed significant upregulation of VEGF-A, KDR, and FLT1 messenger RNA on day 86 in the MB-PlGF group $(P<.05$; Figure $4, J-L)$ relative to sham, control, and MB groups. PlGF messenger RNA expression (Figure 4, $M$ ) and VEGF-A protein expression (Figure $4, N$ ) were the same in all experimental groups. PlGF protein expression was significantly increased in the $\mathrm{MB}$ and MP-PIGF groups relative to the sham and control groups $(P<.05$; Figure $4, O)$. These results indicate that PlGF enhances angiogenesis.

\section{PIGF Affects Myocardial MMP and TIMP Expressions}

On day 86 after myocardial infarction, protein expressions of the key angiogenic proteases MMP-2 and MMP-9 were significantly upregulated in the MB-PIGF group relative to all other groups (Figure 5, $A$ and $B$ ). In addition, significant increases in TIMP-1 and TIMP -2 expressions were seen in the MB-PlGF group relative to the sham, control, and MB groups (Figure 5, C and D). Myocardial TIMP-3 protein expression was significantly increased in both the MB and MB-PlGF groups relative to the sham and control groups (Figure 5,E), although TIMP-4 was downregulated in sham and both myoblast groups relative to the control group (Figure 5, F). These data indicate that PlGF overexpression enhances the protein expression of angiogenic MMPs and differentially affects the protein expressions of their tissue inhibitors.

\section{DISCUSSION}

In a series of experiments, it has been shown that loss of PIGF impairs pathologic angiogenesis in the ischemic limb and heart without affecting physiologic angiogenesis. ${ }^{10}$ Conversely, PlGF treatment has been shown to stimulate angiogenesis and collateral growth in the ischemic myocardium and limb. ${ }^{15}$ Recently, it has been shown that systemic delivery of PIGF, resulting in elevated circulating levels of PIGF for 4 weeks, can improve cardiac function and stimulates angiogenesis in the infarct border and vessel enlargement in the remote myocardium. ${ }^{16}$ Furthermore, intramyocardial delivery of PlGF after myocardial infarction enhances angiogenesis in the border zone and attenuates adverse ventricular remodeling while preserving cardiac function. ${ }^{17}$ Myoblast transplant into the ischemic heart has been shown to exert beneficial effects after experimental myocardial infarction..$^{1-3}$ Nonetheless, in the first clinical study, myoblast transplant into the ischemic heart failed to improve cardiac function, although distinct antiremodeling effects were achieved. ${ }^{5}$ On the other hand, various experimental studies have indicated that the combination of myoblasts with growth factors can expand the efficiency of myoblast transplant. ${ }^{6,7}$ Our study was therefore designed to create genetically engineered myoblasts overexpressing PIGF and to investigate their role in angiogenesis, myoblast survival, cardiac remodeling, and cardiac function in an experimental heart failure model. The beneficial role of PIGF after acute myocardial infarction is well documented, ${ }^{10,12,15-17}$ and PIGF has been shown to exert positive effects on vessel maturation and vessel durability, influencing endothelial cells, smooth muscle cells, and macrophages. Smooth muscle cells in particular are crucial for arteriogenesis, because vessels covered with smooth muscle cells fail to regress, even when the angiogenic stimulus fades away. ${ }^{12}$

We observed significantly more vWF-positive and $\alpha$ SMA-positive vessels and upregulated VEGF-A, FLT1, and KDR gene expressions in the MB-PlGF group. VEGF-A plays a key role in angiogenesis ${ }^{9}$ and induces blood vessel formation after experimental myocardial infarction. ${ }^{8}$ The VEGF receptors FLT1 and KDR are important for endothelial cell differentiation, migration, proliferation, and angiogenesis. ${ }^{9}$ There were no significant differences in PlGF messenger RNA expression in myocardial tissue among all experimental groups on day 86. PlGF protein expression, however, was increased in both the MB and MB-PIGF groups relative to the sham group. This finding has 2 potential implications. First, acute myocardial infarction per se could have contributed to PIGF overexpression, as previously reported. ${ }^{14}$ Second, because vascular density and cardiac function were improved in the

difference from control and unmodified myoblast groups (G, H, $P<.01$ ). Bar represents $50 \mu \mathrm{m}$ (A, B, E, F) or $100 \mu \mathrm{m}$ (C, D). I, Changes in placental growth factor $(P l G F)$ messenger RNA ( $m R N A)$ levels after transfection of myoblasts with placental growth factor plasmids in vitro relative to controls. Asterisk indicates significant difference from control $(P<.001)$. Quantification of messenger RNA $(m R N A)$ levels of vascular endothelial growth factor A $(V E G F-A, \mathrm{~J})$, placental growth factor $(P l G F, \mathrm{~K})$, fms-like tyrosine kinase $(F l t-1, \mathrm{~L})$, and kinase insert domain receptor $(K D R$, $\mathrm{M})$, and protein levels of vascular endothelial growth factor A $(V E G F-A, \mathrm{~N})$ and placental growth factor $(P l G F, \mathrm{O}) 86$ days after myocardial infarction. Asterisk indicates significant difference from sham, control, and unmodified myoblast groups in $\mathrm{J}(P<.05)$. Asterisk indicates significant difference from sham group and dagger indicates significant difference from control and unmodified myoblast groups in $\mathrm{L}(P<.05)$. Asterisk indicates significant difference from sham, control, and unmodified myoblast groups in $\mathrm{M}(P<.05)$. Asterisk indicates significant difference from sham group in $\mathrm{O}(P<.05)$. LC, Loading control; $S P$, specific protein. 

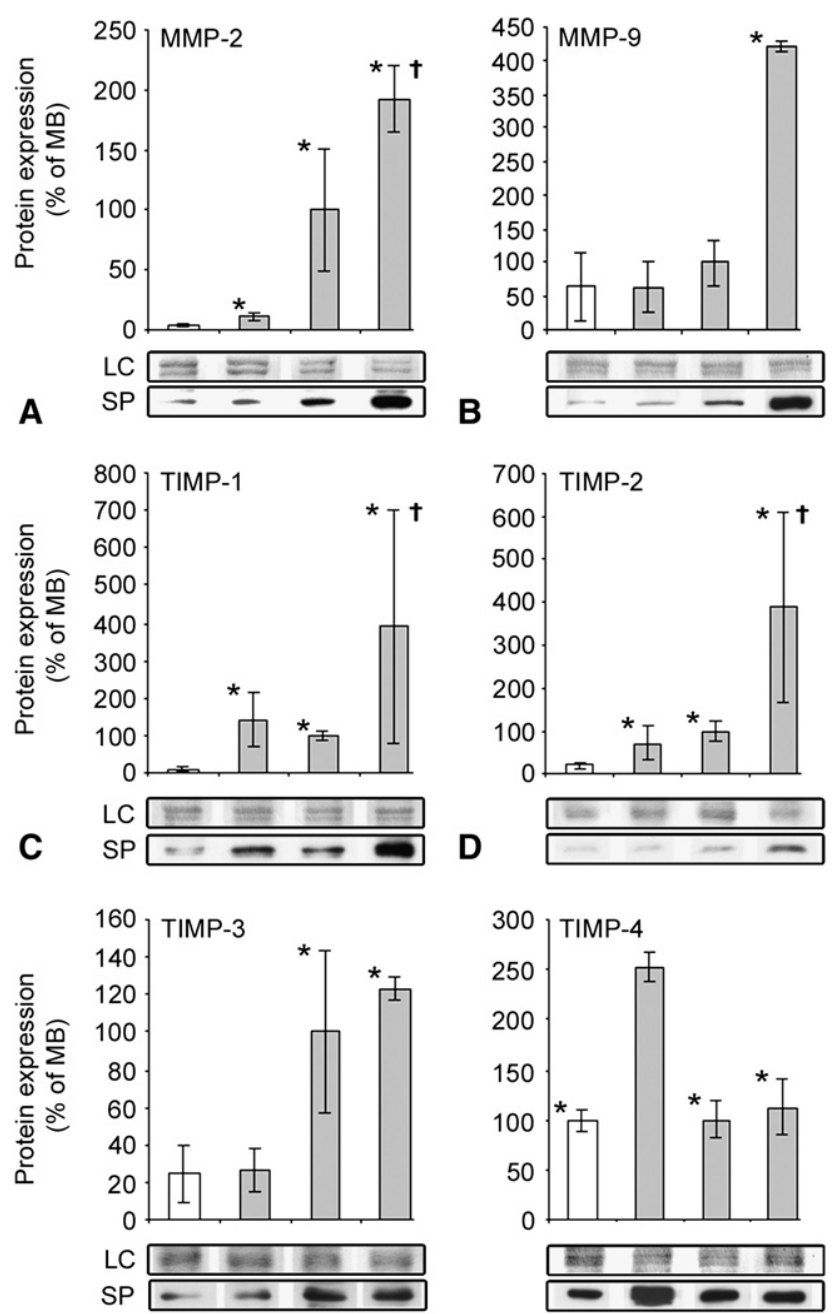

E

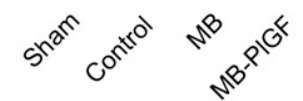

$\mathbf{F}$

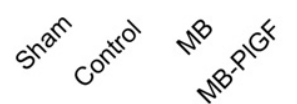

FIGURE 5. Matrix metalloproteinase $(M M P)$ and tissue inhibitor of matrix metalloproteinase (TIMP) protein expressions in sham, control, unmodified myoblast $(M B)$, and transfected myoblast groups $(M B-P l G F)$. Asterisk indicates significant difference from sham group $(P<.05$ for control and unmodified myoblast groups, $P<.001$ for transfected myoblast group) and dagger indicates significant difference from control and unmodified myoblast groups $(P<.001$ for control and $P<.05$ for unmodified myoblasts) in A. Asterisk indicates significant difference from sham, control, and unmodified myoblast groups $(P<.0001)$ in B. Asterisk indicates significant difference from sham group $(P<.01$ for control and unmodified myoblast groups, $P<.05$ for transfected myoblast group) and dagger indicates significant difference from control and unmodified myoblast groups $(P<.05)$ in $\mathrm{C}$. Asterisk indicates significant difference from sham group $(P<.01$ for control group, $P<.001$ for unmodified myoblast group, and $P<.05$ for transfected myoblast group) and dagger indicates significant difference from control and unmodified myoblast groups $(P<.05)$ in D. Asterisk indicates significant difference from sham and control groups $(P<.05$ for unmodified myoblast group vs sham and control groups, $P<.001$ for transfected myoblast group vs sham and control groups) in E. Asterisk indicates significant difference from control group $(P<.001)$ in F. $L C$, Loading control; $S P$, specific protein.
MB-PIGF group but not in the MB group, we assume that transient PIGF overexpression may provide enormous benefits in the repair of infarcted myocardium as result of the prolonged production of angiogenic factors by myoblasts that overexpress PIGF and induce the formation of durable vessels, as shown by our results. This hypothesis is further supported by the fact that in our experimental setting myoblasts transfected with PlGF overexpressed PIGF in vitro for at least 14 days. These results are also in line with data showing that PIGF enhances angiogenesis and arteriogenesis within 7 days in ischemic myocardium. ${ }^{12}$

In addition to angiogenesis, FS was increased relative to baseline levels on days 52 and 86 in the MB-PIGF group. Although higher mean values on days 52 and 86 relative to baseline levels were also found in the MB group, those results did not reach statistical significance. Moreover, FS on day 86 in the MB-PIGF group was significantly improved relative to FS on day 86 in both the MB and control groups. Importantly, on day $86 \mathrm{LV}$ diameters, LV volumes, and consequently EF were significantly improved in the MB-PIGF group. These results clearly demonstrate the beneficial effect of MB-PIGF on cardiac function.

We also investigated the influence of MB-PIGF on myoblast graft survival. It has been reported previously that the outcome of myoblast transplant is dependent on the number of grafted cells, ${ }^{3}$ and our results are in line with this finding. Fluorescent imaging revealed significant differences in the size of the area containing myoblasts between the MB and MB-PlGF groups. In addition, the number of labeled cells was significantly increased in the MB-PlGF group. These results indicate that PIGF exerts beneficial effects on myoblast survival that positively contribute to angiogenesis and cardiac function. In this study, we did not perform additional staining for surviving myoblasts; however, our previous work clearly points to a high correlation between DiIC $_{18}$ fluorescence and skeletal muscle myosin heavy chain staining. ${ }^{6}$

Although we found a significant reduction in LV fibrosis in both the MB and MB-PIGF groups relative to the control group, the largest LV infarct wall thickness was seen in the MB-PlGF group. In this study, unmodified myoblasts reduced cardiac fibrosis, but our results also indicate that these antiremodeling effects of unmodified myoblasts cannot contribute to the improvement of cardiac function, LV infarct wall thickness, or enhanced vessel formation. MMPs and TIMPs are involved in extracellular matrix turnover in both physiologic and pathologic situations. ${ }^{18}$ It has been reported that MMPs are increased after acute myocardial infarction and that MMP inhibitors beneficially affect cardiac remodeling and function, ${ }^{18}$ although another study indicates that prolonged MMP inhibition does not prevent LV remodeling and dysfunction but rather impairs postinfarct angiogenesis, ${ }^{19}$ associated with higher mortality. ${ }^{20}$ An excessive fibrosis in the absence of contractility or 
relaxation, however, could accelerate cardiac remodeling and decrease cardiac function. On this basis, increased expression of MMPs and proteolysis of excess collagen may be a protective mechanism in chronic myocardial infarction. Myoblasts in combination with relaxin are reported to upregulate MMP-2, MMP-9, and VEGF expressions, resulting in enhanced angiogenesis and improved cardiac function. ${ }^{21}$ In concert with these findings, our treatment protocol resulted in overexpression of both MMP-2 and MMP-9, enzymes that reportedly mediate angiogenesis. ${ }^{21-23}$ The myocardial MMP profile in the MB-PIGF group therefore suggests that myoblasts transfected with PlGF positively influence cardiac tissue remodeling through MMP-2 and MMP-9 upregulation. This effect may also comediate PlGF-induced angiogenesis, because that process is dependent on degradation of extracellular matrix components. Finally, the delivery of the PlGF gene by myoblast transplant resulted in differentially expressed TIMPs. The reason for this phenomenon is unclear; however, earlier results on the positive effects of TIMP-3 on cardiac remodeling in a knockout model ${ }^{24}$ may point to the contribution of this enzyme, at least in part, to the observed reverse cardiac remodeling.

In sum, we have demonstrated that PIGF-transfected myoblasts induce angiogenesis, enhance myoblast graft survival, improve cardiac function, and ameliorate cardiac remodeling. This approach may be beneficial in the treatment of chronic heart failure. As a limitation of this study, however, we acknowledge that fluorescent imaging of surviving myoblasts, even though fluorescent cells have been detected in tissue lysates and quantified, might not be as powerful as detection of chromosomes by transplanting myoblasts in sex-mismatched groups. Also, although our findings were statistically significant, the fact that not all animals were studied with in vivo imaging and fluorescent microscopy may have had some effect on the results that was not accounted for in data interpretation.

\section{References}

1. Menasché P, Hagège AA, Vilquin JT, Desnos M, Abergel E, Pouzet B, et al. Autologous skeletal myoblast transplantation for severe postinfarction left ventricular dysfunction. J Am Coll Cardiol. 2003;41:1078-83.

2. Jain M, DerSimonian H, Brenner DA, Ngoy S, Teller P, Edge AS, et al. Cell therapy attenuates deleterious ventricular remodeling and improves cardiac performance after myocardial infarction. Circulation. 2001;103:1920-7.

3. Tambara K, Sakakibara Y, Sakaguchi G, Lu F, Premaratne GU, Lin X, et al. Transplanted skeletal myoblasts can fully replace the infarcted myocardium when they survive in the host in large numbers. Circulation. 2003;108. Suppl. 1:II259-63.

4. Hagège AA, Marolleau JP, Vilquin JT, Alhèritiére A, Peyrard S, Duboc D, et al. Skeletal myoblast transplantation in ischemic heart failure: long-term follow-up of the first phase I cohort of patients. Circulation. 2006;114(1 Suppl):I108-13.

5. Menasché P, Alfieri O, Janssens S, McKenna W, Reichenspurner H, Trinquart L, et al. The Myoblast Autologous Grafting in Ischemic Cardiomyopathy (MAGIC) trial: first randomized placebo-controlled study of myoblast transplantation. Circulation. 2008;117:1189-200.

6. Aharinejad S, Abraham D, Paulus P, Zins K, Hofmann M, Michlits W, et al. Colony-stimulating factor-1 transfection of myoblasts improves the repair of failing myocardium following autologous myoblast transplantation. Cardiovasc Res. 2008;79:395-404.

7. Azarnoush K, Maurel A, Sebbah L, Carrion C, Bissery A, Mandet C, et al. Enhancement of the functional benefits of skeletal myoblast transplantation by means of coadministration of hypoxia-inducible factor $1 \alpha$. J Thorac Cardiovasc Surg. 2005;130:173-9.

8. Ye L, Haider HKh, Tan R, Toh W, Law PK, Tan W, et al. Transplantation of nanoparticle transfected skeletal myoblasts overexpressing vascular endothelial growth factor-165 for cardiac repair. Circulation. 2007;116(11 Suppl): I113-20.

9. Tammela T, Enholm B, Alitalo K, Paavonen K. The biology of vascular endothelial growth factors. Cardiovasc Res. 2005;65:550-63.

10. Carmeliet P, Moons L, Luttun A, Vincenti V, Compernolle V, De Mol M, et al Synergism between vascular endothelial growth factor and placental growth factor contributes to angiogenesis and plasma extravasation in pathological conditions. Nat Med. 2001;7:575-83.

11. Nagy JA, Dvorak AM, Dvorak HF. VEGF-A(164/165) and PlGF: roles in angiogenesis and arteriogenesis. Trends Cardiovasc Med. 2003;13:169-75.

12. Luttun A, Tjwa M, Moons L, Wu Y, Angelillo-Scherrer A, Liao F, et al. Revascularization of ischemic tissues by PlGF treatment, and inhibition of tumor angiogenesis, arthritis and atherosclerosis by anti-Flt1. Nat Med. 2002;8:831-40.

13. DiSalvo J, Bayne ML, Conn G, Kwok PW, Trivedi PG, Soderman DD, et al Purification and characterization of a naturally occurring vascular endothelial growth factor placenta growth factor heterodimer. J Biol Chem. 1995;270: 7717-23.

14. Iwama H, Uemura S, Naya N, Imagawa K, Takemoto Y, Asai O, et al. Cardiac expression of placental growth factor predicts the improvement of chronic phase left ventricular function in patients with acute myocardial infarction. $J$ Am Coll Cardiol. 2006;47:1559-67.

15. Luttun A, Tjwa M, Carmeliet P. Placental growth factor (PIGF) and its receptor Flt-1 (VEGFR-1): novel therapeutic targets for angiogenic disorders. Ann N Y Acad Sci. 2002;979:80-93.

16. Roncal C, Buysschaert I, Chorianopoulos E, Georgiadou M, Meilhac O, Demol $\mathrm{M}$, et al. Beneficial effects of prolonged systemic administration of PlGF on late outcome of post-ischaemic myocardial performance. J Pathol. 2008;216:236-44.

17. Kolakowski S Jr, Berry MF, Atluri P, Grand T, Fisher O, Moise MA, et al Placental growth factor provides a novel local angiogenic therapy for ischemic cardiomyopathy. J Card Surg. 2006;21:559-64.

18. Vanhoutte D, Schellings M, Pinto Y, Heymans S. Relevance of matrix metalloproteinases and their inhibitors after myocardial infarction: a temporal and spatial window. Cardiovasc Res. 2006;69:604-13.

19. Tessone A, Feinberg MS, Barbash IM, Reich R, Holbova R, Richmann M, et al. Effect of matrix metalloproteinase inhibition by doxycycline on myocardial healing and remodeling after myocardial infarction. Cardiovasc Drugs Ther. 2005; 19:383-90.

20. Spinale FG, Escobar GP, Hendrick JW, Clark LL, Camens SS, Mingoia JP, et al Chronic matrix metalloproteinase inhibition following myocardial infarction in mice: differential effects on short and long-term survival. $J$ Pharmacol Exp Ther. 2006;318:966-73.

21. Formigli L, Perna AM, Meacci E, Cinci L, Margheri M, Nistri S, et al. Paracrine effects of transplanted myoblasts and relaxin on post-infarction heart remodelling. J Cell Mol Med. 2007;11:1087-100.

22. Cheng XW, Kuzuya M, Nakamura K, Maeda K, Tsuzuki M, Kim W, et al. Mechanisms underlying the impairment of ischemia-induced neovascularization in matrix metalloproteinase 2-deficient mice. Circ Res. 2007;100:904-13.

23. Johnson C, Sung HJ, Lessner SM, Fini ME, Galis ZS. Matrix metalloproteinase-9 is required for adequate angiogenic revascularization of ischemic tissues: potential role in capillary branching. Circ Res. 2004;94:262-8.

24. Kassiri Z, Oudit GY, Sanchez O, Dawood F, Mohammed FF, Nuttall RK, et al. Combination of tumor necrosis factor-alpha ablation and matrix metalloproteinase inhibition prevents heart failure after pressure overload in tissue inhibitor of metalloproteinase-3 knock-out mice. Circ Res. 2005;97:380-90. 